\title{
Untersuchungen über Merkmale für die Eineiigkeitsdiagnostik bei Rinderzwillingen
}

\section{Doctoral Thesis}

Author(s):

Nadai, Josef

Publication date:

1949

Permanent link:

https://doi.org/10.3929/ethz-a-000092058

Rights / license:

In Copyright - Non-Commercial Use Permitted 
Diss. Eтн: $1676 \beta$.

\title{
Untersuchungen über Merkmale für die Eineiigkeitsdiagnostik bei Rinderzwillingen
}

\author{
Von der \\ Eidgenössischen Technischen Hochschule in Zürich \\ zur Erlangung \\ der Würde eines Doktors der Technischen Wissenschaften \\ genehmigte \\ Promotionsarbeit
}

\author{
Vorgelegt von \\ Josef Nadai, dipl. ing. agr. \\ aus Cegléd (Ungarn)
}

Referent: Herr Prof. Dr. H. Lörtsch er

Korreferent: Herr Prof. Dr. J. Ri eder

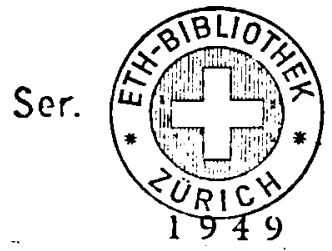

HERMANN BEYER \& SOHNE (BEYER \& MANN), LANGENSALZA 
Bei Annahme der Zweieigkeit wären allerdings Übereinstimmungen von der Art der zwischen den Zeichnungen der Köpfe (Augenbogen) bestehenden kaum zu erklären, es sei denn, die im Bestande des Züchters der Zwillinge herrschende Inzucht würde hierfür verantwortlich erklärt werden. Die Zwillinge gehören nämlich einer Herde der schweizerischen Schwarzfleckviehrasse an, die sich, vollkommen isoliert, inmitten eines ausgesprochenen Braunviehzuchtgebietes befindet. Zufolge der Schwierigkeiten in der Beschaffung des männlichen Zuchtmaterials hat im Bestande schon seit langem eine mehr oder minder intensive Inzucht Platz gegriffen. Über deren Ausma8 können, mangels einer Zuchtbuchführung nur Vermutungen und Schätzungen angestellt werden, doch könnte. sie zweifellos zur Erklärung der hochgradigen Übereinstimmung in den Pigmentationszentren der Zwillinge mit herangezogen werden.

Die diagnostische Bewertung der Flotzmaulabdrücke des Paares Trudy/Friedy trifft auf ganz ähnliche Hindernisse wie jene der Scheckung. Da zur Zeit nichts Sicheres darüber bekannt ist, ob zwischen Linienverlauf der Papillarfelder und Charakter der Papillarelemente erbliche Korrelationen bestehen, ist es nicht möglich zu entscheiden, ob die Differenzen im Papillarcharakter ein Ausdruck derselben stark umweltlabilen Erbanlage sind oder auf die Wirkung alleler Gene zurückgeführt werden müssen. Während der erstere Fall eine Eineiigkeit zulieBe, verböte.der zweite eine solche Annahme

Die Parallelität zwischen den Verhältnissen beim Flotzmaul und bei der Scheckung ist im vorliegenden Falle um so interessanter, als es sich bei beiden um Merkmale handelt, die eng mit der Hautbeschaffenheit in Verbindung stehen. Bei Friedy verbindet sich starke Schwarzfärbung des Kopfes mit grober Papillarzeichnung des Flotzmaules, bei Trudy $s c h w a c h$ e Färbung des Kopfes mit f ein en Papillarlinien. Sollten beide Erscheinungen ursächlich verbunden sein?

Die Zwillinge Trudy/Friedy dürfen nach den vorliegenden Verhältnissen jedenfalls. nur als möglicherweise einei ig bezeichnet werden.

\section{Zusammenfassung}

Einleitend wird es als Zweck der vorliegenden Arbeit bezeichnet, die bis anhin in der Zwillingsforschung beim Rind aufgebaute Ähnlichkeitsdiagnostik auf Grund eigener Erfahrungen kritisch zu beleuchten und nach etwaigen Verbesserungsmöglichkeiten $\mathrm{zu}$ suchen.

In der $\mathrm{L}$ it e rat u r übersicht werden anhand der wichtigsten einschlägigen Arbeiten der derzeitige Stand der Zwillingsforschung beim Rind und der Stand der Eineiigkeitsdiagnose bei Rinderzwillingen dargestellt. Als wichtigste neuere Entwicklungstendenzen werden genannt: I. eine Reduktion der ursprünglich bedeutenden Zahl der KronAcherschen Ähnlichkeitsmerkmale (22) auf einige wenige, durch die späteren Zwillingsuntersucher (SCHMIDT, BonNIER, DRY), 2. die Ausschaltung der physiologischen Ähnlichkeitsmerkmale zugunsten der morphologischen und 3. das Fallenlassen der KroNacherschen Ahnlichkeitsbewertung anhand des Ähnlichkeitsquotienten.

Als Anforderungen an eine praktische Eineiigkeitsdiagnostik beim Rind werden genannt und in ihren theoretischen Grundlagen diskutiert: I. die möglichst große Zahl der berücksichtigten Ähnlichkeitsmerkmale, 2. die möglichst geringe Beeinflussung der peristatischen Variabilität der Ähnlichkeitsmerkmale, 3. die voneinander unabhängige Vererbung der Ähnlichkeitskriterien, 4. die möglichst große erbliche Variation der Ähnlichkeitskriterien innerhalb der. Vergleichspopulation, 5. die polymere Vererbung der Änlichkeitsmerkmale, 6. das Unbeeinflußtbleiben von der Zuchtwahl, 7. die möglichst einfache Registrierbarkeit der Ähnlichkeitsmerkmale, 8. die objektive Bewertbarkeit der Änlichkeitskriterien und 9. die zeitlich bequeme Handhabung der ganzen Eineiigkeitsdiagnose. 
Im Bericht über die eigenen Untersuchungen werden die folgenden Ähnlichkeitskriterien angeführt und auf Grund eigener Erfahrungen kritisch besprochen:

I. Die ä u $B$ e e Erschein ung: Es wird festgestellt, daß dieser Merkmalskomplex, der als Hauptkomponenten den Körperbau, die Haarfarbe, die Scheckung und Abzeichen umfaßit, gesamthaft, als e i n Merkmal betrachtet, den Anforderungen an ein Ahnlichkeitskriterium in hohem Maße gerecht wird und als wertvoller: Hinweis auf das Vorliegen oder Fehlen von Eineigkeit betrachtet werden muß.

2. Di e Körpermaße: Diesem Ähnlichkeitskriterium wurde in den Arbeiten Kronachers und Schmidts (HaAk) größte Bedeutung zugernessen. Es wird gezeigt, daß die Körpermessungen, bei aller scheinbaren Präzision, in ihrer Brauchbarkeit zur Eineiigkeitsdiagnose nicht überschätzt werden dürfen und nur in gewissen extremen Fällen von großer Ubereinstimmung, bzw. Verschiedenheit, eindeutige Hinweise zu geben imstande sind.

3. Der Flotzmaulabdruck: Er wird als eines der Fundamentalkriterien bezeichnet, wozu ihn vor, allem die große individuelle Variation und die postnatale Umweltstabilität befähigen. Es wird ein Mangel an objektiven Grundlagen beim Vergleich der Partnerabdrücke jestgestellt und ein eigenes Verfahren zur Auszählung der Papillarelemente beschrieben; das geeignet erscheint, dem Partnervergleich eine gewisse Präzision zu verleihen.

4. Der Augenhintergrund: Als ein dei menschlichen Zwillingsforschung entliehenes Ähnlichkeitskriterium wird der Augenhintergrund auf seine Brauchbarkeit hin theoretisch diskutiert und festgestellt, daß von den verschiedenen Fundusmerkmalen diagnostisch vor allem die Fundusfarbe und -fleckung, sowie die Papille zu beachten sind. Es werden die freie Beobachtung des Augenhintergrundes beim Rind geschildert und verschiedene, zum Teil auch selbst erprobte, mit einfachen Mitteln arbeitende Verfahren der Fundusphotographie beschrieben und kritisch gewürdigt.

5. Die $\mathrm{H}$ a a rwirbe l: Von den neueren Zwillingsuntersuchern (BonNIER, DRY) zum Fundamentalkriterium erhoben, haftet den Haarwirbeln nach den eigenen Erfahrungen der Nachteil einer beschränkten individuellen Variation an, weshalb sie nur in Fällen abnormer Anordnung und Beschaffenheit von diagnostischem Interesse sein können.

6. Die Afterzitzen : Ihre diagnostische Auswertung wird vorderhand durch den Umstand erschwert, daß noch keine sichere Unterscheidung zwischen erblichen und nichterblichen Formen, deren gleichzeitiges Nebeneinanderbestehen von verschiedenen Autoren behauptet wird, getroffen werden kann.

7. Der Erythrozytendurchmes'ser: Im Sinne eines Tastversuches wird bei einem eineiigen Zwillingspaar die Bestimmung der Erythrozytendurchmesser vorgenommen und dabei hochgradige Partnerähnlichkeit festgestellt, woraus aber ohne vorherige Prüfung eines größeren Materials keine endgültigen Schlüsse gezogen werden dürfen.

8. Die Blutgruppen: Die neueren Untersuchungen amerikanischer Forscher haben. Ergebnisse zutage gefördert, welche die Blutfaktoren des Rindes im Rahmen der Eineiigkeitsdiagnose als höchst bedeutsam erscheinen ließen, wenn nicht zufolge Queranastomosenbildung der 
Choriongefäße bei über neun Zehntel der Zwillingsföten während des intrauterinen Lebens eine Angleichung der Bluteigenschaften einträte. Die Blutfaktorenbestimmung hat dementsprechend nur in jenen ro \% der Fälle einen Sinn, wo zwischen den Embryonalhüllen keine Gefäßverbindung bestand. Die eigenen Blutgruppenuntersuchungen beim Rind bestätigen die Ergebnisse früherer Untersuchungen.

9. Die Milchleistungen: Milchleistungserhebungen werden zufolge verschiedener Umstände (Beschränkung auf ein Geschlecht, späte Manifestation, lange Erhebungsdauer usw.) nur in Ausnahmefällen als diagnostische Merkmale in Frage kommen. Die Untersuchungen zeigen, dab dem Milchfettgehalt und gewissen anderen Größen des Milchchemismus der diagnostische Vorzug vor der absoluten Milchmengeleistung gebührt. Als diagnostisch wertvoll erweist sich der Vergleich von Laktationskurven.

Io. Die Hauttransplantation: Versuchsweise wird an einem eineiigen $Z$ willingspaar eine Hauttransplantation durchgeführt, wobei zwischen den beiden Partnern in der Schultergegend zwei Hautlappen ausgetauscht werden (Homöotransplantation) und gleichzeitig zur Kontrolle ein Hautlappen von einem fremden Tier auf den einen Zwillingspartner übertragen wird (,Heterotransplantation"). Der Erfolg ist negativ, indem alle drei Lappen nach vorübergehender Anheilung in kürzeren Intervallen abgestoßen werden.

II. B lutphysiologische Werte: An Blutproben eines eineiigen $Z$ willingspaares werden einige physiologische Werte (Erythrozytenzahl, Hämoglobingehalt usw.) bestimmt. Die Ergebnisse bestätigen die Vermutung, daß physiologische Blutmerkmale in hohem Maße umweltlabil sind und veranschaulichen deutlich, weshalb derartige Merkmale in den Eineiigkeitsdiagnosen der neueren Untersucher mehr und mehr unberücksichtigt blieben.

Abschließend werden drei Beis piele von Eineiigkeitsd i a $g$ n o s e $n$ ausführlich besprochen, nämlich ein Fall von kennzeichnend zweieiigen Zwillingen und ein solcher von kennzeichnend eineiigen, welche beiden Fälle diagnostisch klar und eindeutig entschieden werden können, sowie ein Grenzfall, anhand dessen gezeigt wird, wie die Beschränktheit der derzeitigen haustiergenetischen Kenntnisse mitunter die Stellung einer eindeutigen Diagnose verunmöglicht.

\section{Schrifttum}

x. Appenzeller, W., Eine einfache Methode zur Besichtigung des Augenhintergrundes ohne Spiegel und ihre Brauchbarkeit beim Pferd, verglichen mit den üblichen Methoden. Arch. f. wiss. u. prakt. Tierheilkde. 72, Heft 6, 1938.

2. BAutr, K. H., Homoiotransplantation von Epidermis bei eineiigen Zwillingen. BRUNS Beitr. zur klin. Chir. 141, Heft 1, I927.

3. Beckershaus, E., Úber eineiige Zwillinge. 'Ztschr. f. Augenheilkunde 91, I922.

4. BELIç, M., Zwillings- und Drillingsuntersuchungen beim Rind unter besonderer Berücksichtigung der Skelettvariabilität. Ztschr. f. Züchtung 49, Heft I, r940.

5. Bonnevie, K., Manifestierung und Rolle der Epidermispolster in der Entwicklung der Papillarmuster. Handbuch d. Erbbiologie d. Menschen 1. Berlin 1940.

6. Bonnier, G., Studies on monozygous cattle twins. Acta Agriculturae Suecana 1, Stockholm 1946.

7. - - and SKARMAN, Sv., Aids to the identification of monozygotic twins in cattle. J. of Hered. Vol. 29, No. 7, 1938.

-8. Briquet, R. and Lush, J. L., Heritability of amount of spotting in HolsteinFriesian catlle. J. of Hered. Vol. 38, No. 4, 1947. 